Revue Gouvernance

Governance Review

\title{
Les organismes centraux fédéraux du Canada comme constructions sociales : représentations des analystes centraux
}

\section{Marc-Antoine Therrien}

Volume 17, numéro 1, 2020

Varia

URI : https://id.erudit.org/iderudit/1070344ar

DOI : https://doi.org/10.7202/1070344ar

Aller au sommaire du numéro

Éditeur(s)

Centre d'études en gouvernance de l'Université d'Ottawa

ISSN

1912-0362 (numérique)

Découvrir la revue

Citer cet article

Therrien, M.-A. (2020). Les organismes centraux fédéraux du Canada comme constructions sociales : représentations des analystes centraux. Revue Gouvernance / Governance Review, 17(1), 88-110.

https://doi.org/10.7202/1070344ar

\section{Résumé de l'article}

L'article explore les représentations des analystes évoluant au sein des organismes centraux. De prime abord perçus comme mystérieux, puissants et irréfutables, les organismes centraux apparaissent, par le biais des récits de l'expérience des participants, comme des organisations fédérales uniques, du fait de leur horizontalité et d'un certain contournement de la logique bureaucratique. Contrairement à leurs interlocuteurs dans les ministères d'exécution, les analystes centraux interagissent avec des " clients ministériels " plus expérimentés et hiérarchiquement plus élevés qu’eux. L'influence disproportionnée qu'ils exercent sur ces derniers est paradoxale dans la mesure où ce sont souvent de jeunes analystes, fraîchement recrutés de l'université, qui se retrouvent à exercer les responsabilités importantes qui incombent aux acteurs des organismes centraux. La dimension humaine de l'expérience au centre suggère, dès le recrutement des analystes, des milieux de travail très exigeants, où les injonctions au dépassement de soi et à l'atteinte des résultats à tout prix induisent du stress pour les analystes, qui sont appelés à interagir régulièrement avec les dirigeants et les acteurs politiques clés du gouvernement fédéral. Le recours à la phénoménologie herméneutique et un appareillage méthodologique approprié à la recherche qualitative permettent de poser un regard neuf sur les organismes centraux en mettant l'accent sur l'expérience intersubjective des analystes et de leurs interlocuteurs. En accord avec la perspective de la construction sociale, selon laquelle le monde social, et par extension les organisations, est issu des interactions entre les individus producteurs de sens et de signification, l'expérience des analystes permet de mieux comprendre les organismes centraux en tant que systèmes sociaux spécifiques. 


\section{Les organismes centraux fédéraux du Canada comme constructions sociales : représentations des analystes centraux}

Par Marc-Antoine Therrien ${ }^{1}$

\section{RÉSUMÉ}

L'article explore les représentations des analystes évoluant au sein des organismes centraux. De prime abord perçus comme mystérieux, puissants et irréfutables, les organismes centraux apparaissent, par le biais des récits de l'expérience des participants, comme des organisations fédérales uniques, du fait de leur horizontalité et d'un certain contournement de la logique bureaucratique. Contrairement à leurs interlocuteurs dans les ministères d'exécution, les analystes centraux interagissent avec des «clients ministériels » plus expérimentés et hiérarchiquement plus élevés qu'eux. L'influence disproportionnée qu'ils exercent sur ces derniers est paradoxale dans la mesure où ce sont souvent de jeunes analystes, fraîchement recrutés de l'université, qui se retrouvent à exercer les responsabilités importantes qui incombent aux acteurs des organismes centraux. La dimension humaine de l'expérience au centre suggère, dès le recrutement des analystes, des milieux de travail très exigeants, où les injonctions au dépassement de soi et à l'atteinte des résultats à tout prix induisent du stress pour les analystes, qui sont appelés à interagir régulièrement avec les dirigeants et les acteurs politiques clés du gouvernement fédéral. Le recours à la phénoménologie herméneutique et un appareillage méthodologique approprié à la recherche qualitative permettent de poser un regard neuf sur les organismes centraux en mettant l'accent sur l'expérience intersubjective des analystes et de leurs interlocuteurs. En accord avec la perspective de la construction sociale, selon laquelle le monde social, et par extension les organisations, est issu des interactions entre les individus producteurs de sens et de signification, l'expérience des analystes permet de mieux comprendre les organismes centraux en tant que systèmes sociaux spécifiques.

Mots clés: organismes centraux, représentations, analystes, identité, phénoménologie herméneutique, construction sociale

\section{SUMMARY}

The article explores how analysts working within central agencies perceive their own organization. At first glance perceived as mysterious, powerful and irrefutable, central agencies appear, through the accounts of the participants' experience, as unique federal organizations,

1. Marc-Antoine Therrien est analyste au gouvernement fédéral et détenteur d'un doctorat en administration publique de l'École nationale d'administration publique à Montréal. marc-antoine.therrien@enap.ca 
due to their horizontality and a certain circumvention of the bureaucratic logic. Unlike their counterparts in line departments, central agency analysts interact with more experienced and hierarchically superior "departmental clients". The disproportionate influence they exert on the latter is paradoxical insofar as it is often young analysts, freshly recruited from university programs, who find themselves exercising these important central agency responsibilities. The human dimension of the experience at the center suggests, right from recruiting stages, very demanding work environments, where injunctions for surpassing oneself and achieving results at all costs induce stress for analysts, who are called upon to interact regularly with senior leaders and key political players in the federal government. The use of hermeneutical phenomenology and an appropriate methodological apparatus for qualitative research allow us to take a fresh look at central agencies by emphasizing the intersubjective experience of analysts and their interlocutors. In accordance with the social construction perspective, according to which the social world, and by extension organizations, stems from the interactions between individuals who are sense makers and meaning producers, the analysts' experience makes it possible to understand central agencies as specific social systems.

Key words : central agencies, perceptions, analysts, identity, hermeneutic phenomenology, social construction

\section{Introduction}

Au cour du gouvernement fédéral canadien se trouvent le Bureau du Conseil privé, le Secrétariat du Conseil du trésor et le ministère des Finances, trois organismes centraux dont le rôle consiste à régir tant les ressources financières que les orientations politiques. En plus de leur centralité au sein de l'appareil administratif, leur positionnement à l'interface politico-administrative leur confère une autorité que redoutent les ministères d'exécution. Le centre demeure peu connu des fonctionnaires et de la population générale.

Cette inaccessibilité du centre se répercute également dans le milieu de la recherche, où prédomine un intérêt pour les structures et les fonctions des organismes centraux. Ainsi, sauf exception (Campbell et Szablowski, 1979; Savoie, 1999), rarement a-t-on accès aux acteurs des organismes centraux et encore moins à l'expérience (inter)subjective qu'ils font du centre. Or, pour quiconque cherche à comprendre le centre, ce regard de l'intérieur est d'une grande richesse, car il permet d'aller au-delà des considérations structuro-fonctionnalistes pour appréhender les organismes centraux comme constructions sociales. Le paradigme de la construction sociale se nourrit des enjeux liés à l'identité sociale et des interactions qui permettent de la façonner, tant sur le plan individuel qu'organisationnel. Une telle perspective favorise ainsi l'acteur dans toute sa 
complexité, soulignant le caractère processuel et récursif de la construction identitaire en milieu organisationnel. Comprendre l'intersubjectivité de l'acteur permet alors de comprendre l'organisation, car l'un et l'autre s'habilitent et se contraignent.

Cet article s'inscrit dans une recherche plus large portant sur la construction identitaire des analystes centraux. L'intérêt pour les représentations des organismes centraux réside dans le rôle qu'elles jouent dans la démarche d'identification et de socialisation organisationnelles de ces derniers, dès leur arrivée au centre. Après avoir brièvement survolé les écrits sur le centre ainsi que l'identité sociale et présenté la méthodologie, j'aborderai ces représentations du centre, selon diverses dimensions qui ont émergé durant l'analyse des entretiens.

\section{Survol des écrits sur le centre au Canada}

Conceptuellement, le centre et les organismes centraux se déclinent de diverses façons, variant selon les dimensions incluses (politique; administrative), le type d'acteurs impliqués (individus; organisations) et les organismes considérés (voir notamment Dunn, 2010; Lindquist, 1996; Savoie, 1997; White, 2005). Nonobstant ces variantes, l'idée demeure que le centre désigne l'«ensemble des organes politiques et des entités administratives de l'État responsables de déterminer les priorités et chargés de la coordination et du contrôle de l'activité gouvernementale» (Bernier et Fortier, 2014, p. 259). Pour les besoins de cet article, je choisis de me concentrer sur trois entités administratives: le Bureau du Conseil privé (BCP), le ministère des Finances (FIN) et le Secrétariat du Conseil du trésor (SCT).

Également appréhendés par le prisme de leur fonction budgétaire prépondérante, les organismes centraux remplissent trois fonctions précises: ils contrôlent les totaux, établissent les priorités et recherchent l'efficience (Schick, 2001). Au tournant des années 2000, les «bureaux budgétaires centraux» ont traversé une crise, en raison de la faible croissance économique, de la consolidation des crédits budgétaires permanents et de la réticence des élus à augmenter les revenus fiscaux (2001, p. 11-12). Aux premiers rangs des réformes managériales, les organismes centraux ont vu leurs rôles évoluer graduellement pour devenir à la fois courtiers d'information, arbitres des affaires ministérielles et défenseurs de l'efficacité et de l'efficience dans le déboursement des deniers publics.

Comme objets de recherche, les organismes centraux fédéraux se laissent surtout étudier de l'extérieur, par le biais de la documentation officielle et de témoignages d'anciens hauts fonctionnaires (voir notamment Clark, 1994; Robertson, 1971). Les chercheurs documentent les structures et les responsabilités de ces organismes, 
focalisent leur attention sur les rapports de force (Hicks, 1973) ou l'influence politique des élites administratives (Savoie, 1999), et problématisent, à l'aune du Nouveau Management public, le centre comme un dilemme entre flexibilité et contrôle des ministères d'exécution (Clark et Swain, 2005; Hart, 2014; Veilleux et Savoie, 1988).

Si on accorde une certaine attention aux mandarins, ces hauts-fonctionnaires habitués des cercles d'influence politique, rarement a-t-on donné la parole aux simples analystes évoluant dans les organismes centraux, malgré leur rôle crucial dans les rouages de l'administration publique. Leur statut de "courtiers honnêtes" et impartiaux des activités gouvernementales participe à l'adoption des valeurs d'intégrité, d'équité et de conciliation dans leurs interactions avec les ministères, ce qui requiert de ces analystes une expertise et des compétences spécifiques (Potter, 2000, p. 122).

\subsection{EC : l'univers des analystes centraux fédéraux}

Au sein de la fonction publique fédérale, le travail d'analyste est largement associé à un groupe professionnel particulier, celui des EC, un acronyme qui signifie Économique et services de sciences sociales. Comme son nom l'indique, cette classification professionnelle regroupe tous les postes dont les responsabilités ont notamment trait à:

"(...) l'exécution de sondages, d'études et de projets dans le domaine des sciences sociales (...); la recherche, l'analyse et l'évaluation de l'incidence économique ou sociologique des politiques, des projets et des programmes ministériels ou interministériels; (...) et l'élaboration, l'analyse et l'interprétation de données qualitatives et quantitatives ainsi que de politiques et de recommandations socioéconomiques»(Canada, 2017, en ligne).

Dans l'ensemble du gouvernement fédéral, les fonctionnaires appartenant au groupe EC représentent 7,4\% des effectifs totaux, en hausse depuis 2000. Au sein des organismes centraux, ce groupe d'employés est beaucoup plus important, alors que les EC représentent 18,8\% des effectifs au BCP, 46,6\% au FIN et 22,9\% au SCT ${ }^{2}$. Dans la région de la capitale nationale, le sigle «EC» a une signification particulière, un certain prestige, diront certains, et contribue à la construction des représentations du centre. Au-delà des responsabilités habituellement associées au groupe professionnel EC plus stratégiques et importantes - et du niveau de scolarité nécessaire pour y accéder, dans les organismes centraux, ce groupe est synonyme de perfectionnement et

2. Données provenant de l'Outil de requête automatisé sur la gestion de l'information des personnes du Bureau du dirigeant principal des ressources humaines au Secrétariat du Conseil du trésor du Canada, en date du 19 février 2019. 
d'avancement. Bien qu'elle ne soit pas propre aux organismes centraux, cette idée de reconnaître, de documenter et de sanctionner le progrès des analystes par l'encadrement et le cheminement d'un échelon à l'autre est assurément plus courante au centre.

\subsection{L'identité sociale et sa construction}

Dans les sociétés valorisant le travail rémunéré, la vie organisationnelle et le travail ont un impact significatif sur la façon de se définir et de s'identifier (Du Gay, 1996, dans Lutgen-Sandvik, 2008, p. 98). Les identités et le travail qui s'y rattache jouent un rôle crucial dans le repérage des pensées, des sentiments et des actions des individus dans des contextes situés (Brown, 2017, p. 299). Les travaux de Mead (1934) ont largement influencé, jusqu'à aujourd'hui, la pensée sur l'identité, particulièrement dans son acception sociale, comme issue des interactions: "Identity, then, is the unending and recursiveperceptions of others'perceptions of the self, and the identities of others are constituted through exactly the same processes (...)» (Knights et Clarke, 2017, p. 341). Cette conceptualisation de soi de l'acteur qui se développe par le truchement de l'adhésion à un groupe a une importante portée émotionnelle pour ce dernier (Tajfel, 1974, p. 69).

L'adhésion à un groupe dépend selon Tajfel de la contribution positive que ce groupe a sur l'identité sociale d'un individu, sans laquelle ce dernier tentera de quitter le groupe, ou si ce n'est pas possible, cherchera à réinterpréter les attributs du groupe pour les rendre acceptables ou à modifier la situation grâce à l'action sociale (1974, p. 69-70). Parallèlement, un groupe social sera en mesure de préserver sa contribution positive à l'identité sociale individuelle de ses membres seulement s'il réussit à maintenir son caractère distinct (1974, p. 72). Comme le groupe, l'association individuorganisation s'appuie sur une dynamique structurante, c'est-à-dire que l'action et la structure jouent un double rôle, l'une envers l'autre, de contrainte et d'habilitation (Barley et Tolbert, 1997; Giddens, 1987).

L'identité sociale en contexte professionnel réfère à ce qu'une personne croit être son rôle au travail, incluant les attentes à son égard en regard de ce qu'elle devra accomplir au nom de l'organisation (Ashforth, Rogers, et Corley, 2011, p. 1145). Certains, comme Haslam et Ellemers, vont jusqu'à dire que, sans identité sociale, il ne pourrait y avoir d'organisations ni d'actes d'organiser (2005, p. 87, dans id.). Ceci sous-entend qu'il existe plusieurs niveaux identitaires (par exemple, personnel, relationnel et collectif (Sluss et Ashforth, 2007)). Ainsi, Ashforth et coll. conçoivent trois niveaux d'analyse extra-individuels de la construction identitaire ancrés dans la subjectivité de l'individu. Sur le plan intrasubjectif se trouvent les cognitions individuelles sur 
l'identité («je pense»); celles-ci facilitent en retour l'émergence de cognitions partagées, à un niveau intersubjectif ("nous pensons»); au fil du temps, ce partage cognitif participera à l'institutionnalisation de la réalité organisationnelle ("c'est») (2011, p. 1146).

Cet aspect récursif de l'identité sociale renvoie à l'idée du processus, de quelque chose d'itératif et de fluide. Le travail identitaire désigne cette dimension processuelle, par laquelle l'acteur tente d'atteindre une certaine cohérence entre son identité personnelle et la (ou les) identité(s) sociale(s) du groupe auquel il adhère, travail impliquant à la fois conciliation et influence (Watson, 2009, p. 431). L'étude des représentations que se font les analystes centraux permet une incursion dans les fondements de cet intense travail identitaire qui les mobilisera pendant plusieurs mois suivant leur recrutement. En se représentant le centre, ils anticipent leur rôle professionnel et entrevoient un positionnement identitaire dont la socialisation organisationnelle se chargera de façon plus nette.

\section{Considérations méthodologiques}

Pour comprendre la démarche identitaire des analystes au sein des organismes centraux, je préconise la perspective de la construction sociale, posture soulignant l'intersubjectivité du monde social et l'importance de l'acteur comme producteur de sens et de significations (Jun, 2006). Une telle perspective présuppose la prépondérance des interactions et de la communication active dans la construction de la réalité organisationnelle, y compris dans sa forme institutionnalisée, tout en rappelant son caractère éphémère et évolutif. Les interactions dans la construction sociale servent à la formation et à la négociation des identités des acteurs et de l'organisation. La dimension identitaire de l'acteur mène à la réflexivité, dernière pièce du puzzle de la construction sociale, par laquelle l'acteur examine son rapport aux autres, remet en question ses idées et ses pratiques, forge son identité et module l'organisation au sein de laquelle il évolue (Chia, 1996).

Les représentations que se font les analystes des organismes centraux participent de leur construction identitaire. Comprendre l'identité comme émergeant des interactions souligne le caractère socialement et culturellement situé de l'individualité (Jun, 2006, p. 149). Pareillement, dans le monde organisationnel, les acteurs, par la communication active et les interactions, organisent la réalité. 
Dans ce contexte interactionnel, je retiens le récit comme mode d'accès à la connaissance; véritable moteur de réflexivité, le récit permet de structurer l'expérience subjective des acteurs (Boje, 2003). Pour appréhender le vécu des acteurs sur le terrain, j'adopte la phénoménologie herméneutique, par laquelle on interprète les structures de l'expérience telle que présentée à la conscience humaine (White, 1999, p. 47-48).

Ma stratégie de recherche s'appuie sur deux outils méthodologiques connexes : l'entretien et la rédaction d'un journal de recherche. L'entretien semi-dirigé permet d'établir un dialogue ouvert avec le participant et fournit la marge de manœuvre et la flexibilité nécessaires à l'approfondissement de certains thèmes ou enjeux pertinents à la recherche (Savoie-Zajc, 2009, p. 340). Le journal de recherche est un outil qui sollicite et stimule la réflexivité du chercheur, lui permettant de consigner les éléments épars ayant trait à la collecte de données et en appui à celle-ci (Annink, 2017, p. 4). Par le truchement de l'analyse phénoménologique interprétative, une méthode systématique qui vise à "démêler" les significations contenues dans les propos d'un acteur ou de plusieurs, par le biais d'un processus d'interprétation du texte issu des transcriptions d'entretiens avec ces derniers (Willig, 2001, p. 53), j'ai procédé à l'identification de thèmes et de l'interprétation des données recueillies (Sloan et Bowe, 2014, p. 1295).

Grâce à une sélection de convenance, dix-sept participants (douze analystes centraux actuels et passés, quatre participants ministériels et un informateur) ont été recrutés selon la méthode boule de neige. Des douze analystes centraux, trois étaient issus du BCP, trois provenaient du FIN et six étaient associés au SCT. La collecte des données a eu lieu de mai à décembre 2018.

\section{Résultats}

\subsection{Conception initiale}

Si certains des analystes admettent n'avoir eu aucune connaissance préalable des organismes centraux, d'autres évoquent cette idée de systèmes organisationnels plus efficaces :

I do believe those organizations in terms of functioning, in terms of people doing their job, in terms of people being responsive, they do it nine out of 10 times. Whereas in a department here it does it five out of 10 times. (Intervenante ministérielle) 
Ce contraste entre le centre et les ministères d'exécution évoque cette idée selon laquelle les entreprises privées seraient plus performantes et horizontales que les organisations du secteur public. Tout s'y déroule et s'y accomplit plus rapidement et plus efficacement, comme au centre.

Certaines conceptions initiales apparaissent plus rudimentaires, s'approchant d'une vision naïve et peu nuancée des organismes centraux. Des représentations qui soulignent le caractère mystérieux et puissant des organismes centraux et leur prépondérance:

Ifelt like they're a bit magical, PCO [Privy Council Office, BCP] was this place and I didn't really know what went on there, it was kind of enchanting... I don't know what happens, but I know it's a really cool place to be. And they very rarely lift the veil, so you know that they hold a lot of weight, you know you're supposed to listen to them. And whenever they ask for something, you drop everything else. (Analyste, SCT)

Certains participants ont recours à la métaphore religieuse lorsqu'ils parlent des organismes centraux, pour en souligner le caractère omnipotent et omniscient. Cette représentation est sans doute exagérée, mais elle suggère l'aura mythique entourant les organismes centraux, qui met en lumière une certaine ignorance initiale:

I remember that when somebody said what PCO says it was like the word of God. It was just irrefutable. If someone from PCO said X, Y, or Z well that was the law. (...) you didn't question it. (Intervenante ministérielle)

Cette représentation de grandeur, d'autorité et de mystère a un impact bien réel sur les acteurs dans l'exercice de leurs fonctions, comme l'illustrent les propos d'une analyste au centre, relatant une expérience antérieure dans un ministère d'exécution:

(...) the perception was built through my boss who, every time we had a meeting with Finance or they emailed us questions, we had to drop everything and respond to them and tend to their needs. And he would be very nervous if we had a delayed response, (...) we couldn't show that we were weak, (...) that we didn't know what we were doing. (Analyste, FIN)

Cet extrait souligne l'aspect intersubjectif et interprétatif de la construction des représentations et des croyances en contexte social, et plus spécifiquement du rapport entre le centre et les ministères d'exécution. 
En somme, les organismes centraux apparaissent puissants, autoritaires et opaques. Dans une telle représentation, les acteurs qui évoluent au centre tiennent des propos - analyses, conseils, opinions, directives - irréfutables et indéniables. Le rapport qu'entretiennent donc les fonctionnaires avec le centre est comparable à celui liant les croyants aux dogmes ou aux canons religieux: il implique la foi et l'obéissance. Cette représentation des organismes centraux, manichéenne, hautement imagée et évocatrice, est particulièrement efficace dans le récit des participants; elle leur permet de se positionner, d'annoncer les couleurs pour la suite de leur histoire, et de justifier l'orientation de la transformation identitaire à s'opérer. Confrontés au grandiose et à l'abstrait, à des forces complexes qu'on ne comprend pas bien, il faut vaincre ou périr, un élément narratif très présent dans les propos des analystes évoquant leur expérience au centre, dans un récit mêlant adversité et résilience. Pour la plupart des analystes, cependant, un portrait plus nuancé émerge au fil de l'expérience.

\subsection{Recrutement et carrière}

La spécificité de l'expérience au centre se manifeste avant que les analystes n'y fassent leur entrée, par le biais du recrutement. En effet, le processus menant à l'embauche des analystes au sein de l'un ou l'autre des organismes centraux diffère, selon ces derniers, des processus de dotation habituels au gouvernement fédéral, où on affiche, sur un babillard virtuel, un poste disponible, en indiquant les compétences et l'expérience recherchées, ainsi que les modalités de mise en candidature. Ici, tout se passe par le bouche à oreille:

It is word of mouth, and it is people you know, so biring managers hire people they know in their circle. The top performers they have in their network, that's who they try to recruit. (Analyste, BCP)

Il y a ainsi quelque chose de fortuit et d'officieux dans l'embauche; puisque rien n'est affiché sur le babillard virtuel, il n'y a pour ainsi dire pas de "processus». Aucun engagement ou promesse d'embauche n'est nécessaire, puisqu'il n'y a pas de poste ouvert. Un poste est disponible seulement si on repère une personne apte à l'occuper.

I sent her an email. I said, "If you know of anything, let me know." She's like, "Totally. Send me your CV," and then three days later, I have an interview. (Analyste, BCP)

Un tel mode d'opération accélère considérablement le "processus " et permet de trier les candidats sur le volet, tous référés par des acteurs évoluant déjà dans les organismes centraux ou des cadres supérieurs ayant des contacts dans ces derniers. 
Il importe toutefois de mentionner que cette pratique n'est pas uniforme à tous les secteurs de tous les organismes centraux; on la retrouve beaucoup au BCP, dans une moindre mesure au FIN, et au SCT, surtout dans les secteurs de programmes.

Outre le bouche à oreille, plusieurs des analystes ont été embauchés à la suite de leur participation à un programme de recrutement post-secondaire. Au centre, ce type de programmes permet de cibler de jeunes diplômés du deuxième cycle universitaire de l'une ou l'autre des disciplines des sciences sociales, sauf pour le FIN, qui privilégie les économistes. L'attrait de la jeunesse, synonyme d'énergie, de dynamisme intellectuel et de détermination, est indéniablement un facteur primordial dans l'embauche de nouveaux analystes, selon une participante:

My experience on the TBS [Treasury Board Secretariat, SCT] side, is that our senior management loves young go-getters. (Analyste, SCT)

L'exercice des fonctions d'analyste au sein d'un organisme central requiert une certaine hardiesse, une bonne confiance en soi, beaucoup de volonté et une énergie pure, car il y a toujours à faire au centre, plus rapidement et plus efficacement:

It's a sweatshop. It's a boiler room. People don't go to be an analyst at PCO or even in a program area at TBS for their entire careers. You go to learn, you go to work really hard and have your brain explode, and then go back to a line department somewhere where you do regular work. (Intervenante ministérielle)

The people who work in the center have a lot of energy. They work long hours. They have a lot of balls in the air, usually. And that's sort of where they wanna be. They're not down about it, you know? They're like, "hey, give me more". There were times where I know people were working consistently until ten o'clock at night on like high priority files. (Analyste, SCT)

En contrepartie, les attentes à l'égard des analystes centraux laissent deviner un milieu de travail particulièrement exigeant et intraitable. Au cours du même entretien, cette participante partage une anecdote à cet effet:

And [my director general] said: "Central agency analysts don't ask for overtime compensation", that it's an honor to be bere, "you should feel privileged to be here, you should not be asking for your overtime". 
Le degré d'intensité du travail, vécu nulle part ailleurs dans la fonction publique fédérale, constitue un moment fort du récit: se lancer dans ce tourbillon, pour une période somme toute assez courte pour la plupart, retenir son souffle et plonger, pour mieux en ressortir avec une expérience unique et distinctive. Tous sont conscients qu'ils pourront "monnayer" cette expérience le temps venu pour obtenir le poste désiré dans un ministère d'exécution.

(...) once you leave the organization you can leverage your experience here and everything you've gained to go to a very specific issue area that you're interested in. Or to seek out that promotion would be far easier than if you were just working your way up in an organization. (Analyste, BCP)

Cette expérience, qu'on imagine aussi riche qu'exténuante, nécessite des ressources d'énergie considérables et certaines dispositions cognitives indispensables. Ainsi se représente-t-on les organismes centraux comme des ministères "anormaux", où les acteurs sont soumis à des exigences plus élevées dès les échelons d'entrée.

\subsection{Horizontalité et proximité du politique}

Il ne serait pas incongru de lier l'intensité de l'expérience au centre à l'horizontalité de l'organisation du travail et à la proximité de la sphère politique, deux éléments de la spécificité des organismes centraux que relèvent les participants dans leurs récits. D'une part, les organismes centraux ont de petits effectifs, ce qui semble se refléter dans leurs structures hiérarchiques, en réduisant l'ampleur du cadre exécutif. Le caractère horizontal des organismes centraux amène de ce fait des effectifs moins expérimentés à être confrontés à des dossiers plus stratégiques impliquant des acteurs plus importants, plus rapidement dans leur carrière, et ce, sans considération préalable à l'égard de leurs capacités professionnelles en la matière.

(...) I think I had the classic central agency experience with that because I was doing things that an EC-O4 [analyste junior-intermédiaire] would never get to do, it was really exciting. I was writing a deck for Treasury Board, I was communicating with ADMs [sous-ministres adjoints] and director generals all the time. I was in meetings with the associate secretary [niveau sous-ministre], like this is really exciting work (...). (Analyste, SCT) 
L'horizontalité est en ce sens considérée comme un impératif d'efficacité:

And there's no discrimination between levels. In a bigger department, you really feel the bureaucracy; the idea of being a flat organization is so true here. You are an analyst dealing with a deputy on a regular basis, and there is no bias there; there's no slapping on the wrist, "you got two levels too high", there's none of that here. It's actually imperative to bave a flat organization in order for us to function well, because if there's something the deputy needs to know right away, there's no better way than to just skip two levels and get to the top. (Analyste, BCP)

Cet accès privilégié aux dirigeants de l'organisation et l'absence de cadres intermédiaires entre l'analyste et ces derniers facilitent certes les communications, mais font augmenter le niveau de stress des analystes, dans la mesure où aucun acteur ne joue le rôle de filtre ou de tampon entre ceux-ci et la haute direction, un processus pourtant bénéfique, en ce qu'il protège ou isole les analystes de ces pressions indues.

Corollaire de cette horizontalité dans l'organisation du travail, la proximité de la sphère politique a des effets similaires, sinon amplifiés, sur la charge de travail des analystes et le contexte dans lequel ils sont appelés à l'effectuer. Un tel contexte est empreint de l'urgence des commandes et de leurs échéanciers, des changements de cap précipités, teintés des exigences et des pressions du politique.

I think there's also a particular environment or management style that stems from our proximity to political actors and how unpredictable and volatile that can mean in terms of the work sometimes. (Analyste, BCP)

Dans quelle mesure la présence du politique, insufflant imprévisibilité et volatilité dans le travail des analystes, témoigne-t-elle de la véritable importance des enjeux? Une participante y réfléchit en ces termes:

(...) then getting to a central agency, the stakes are so much higher. And I don't know if they actually are higher and our roles are more important, or if it's trumped up. I definitely think this comes from a secretary, an assistant secretary who are like that (snaps fingers) with ministers weekly and having to please them. And so that pressure, it just trickles down. If we don't deliver, their jobs are on the line. You come to work every day, you feel that pressure, you feel that responsibility. (Analyste, SCT) 
La proximité de la sphère politique requiert également des analystes qu'ils adaptent leurs produits (analyses, conseils, recommandations, mémos, etc.) pour un lectorat qui n'appartient pas au fonctionnariat, qui n'en a ni le langage, ni les attentes, ni les objectifs. Il s'agit d'un autre univers, pour ainsi dire, et ces analystes évoluent à la rencontre de ces deux univers aux finalités foncièrement distinctes.

Like that's bureaucracy. The programs are bureaucracy. And we're presenting to ministers who have to please their constituents. Who have to go back and say that this money's doing something. Who have to get reelected. (Analyste, SCT)

La négociation entre ces rationalités ou ces logiques institutionnelles parfois divergentes ne se fait pas sans heurt pour les analystes, qui doivent faire preuve de sagacité et d'adaptabilité. S'il est éprouvant, un tel régime de travail, dans lequel les acteurs sont presque continuellement sur le qui-vive, est néanmoins partie intégrante d'une culture organisationnelle axée sur la performance, l'efficacité et un ineffable sentiment de prépondérance dans le déroulement des affaires gouvernementales.

\subsection{Culture organisationnelle}

Plusieurs des participants abordent la culture organisationnelle dans leurs représentations des organismes centraux. Ces cultures fournissent autant d'indicateurs pour les nouveaux venus de ce que signifient appartenance et conduite dans l'organisation.

Tant au sein des organismes centraux que pour les interlocuteurs ministériels, le prestige et la supériorité tiennent de la convention et sont évoqués dès l'arrivée au centre.

I think all the central agencies do this. Finance and Treasury Board have their university recruitment; you do the bootcamps, and they make you look to the person to your left and your right: "You're working with the smartest people in government". They say things like that, right when you enter as a university recruit. [...]

Maybe it's not done in indoctrination. But it's subtleties, it's the day-to-day. It's the way you talk about other people. It's the privilege of information you have. I still remember, everybody always picked up my phone calls when I used to call from Finance. Because you're Finance and this is Finance calling, you get back to them. (Intervenante ministérielle) 
Cette tendance, tant collective qu'individuelle, à cultiver sa superbe et à croire profondément en ses capacités, en sa propre raison d'être et en son pouvoir d'influence n'a rien d'étranger à une certaine culture de dénigrement envers les ministères d'exécution. À l'occasion, on dénigre l'entité entière, parfois, les individus qui la représentent; il s'agit d'un enjeu identitaire, les Autres ("eux») permettant de renforcer le "nous" des analystes centraux. Paradoxalement, les analystes sont très conscients de l'importance d'entretenir des relations harmonieuses avec leurs interlocuteurs ministériels, relations qu'ils souhaitent au demeurant constructives et authentiques, dans la mesure du possible, malgré les craintes qu'ils peuvent susciter et qui sont à la base de leur redoutable efficacité. On dénigre donc à l'interne, entre collègues, dans les réunions d'équipe, et entre collaborateurs des organismes centraux. On peut y voir un facteur culturel de mobilisation et de cohésion.

I always used to feel like I'm working in a better organization and my skillset and my files are more important than... like my work is more valuable. And it's like they ingrain that in you. It's bow the management is. They make fun of the line departments about their capabilities, about the people who work there. [...] This is like water cooler talk. You just make jokes about the rest of government thinking we're a bit perfect. So, you start believing that after a while when you are in that environment. Also when you have repeated interactions with line department that might confirm yourviews, right? (Intervenante ministérielle)

I think this is why central agencies get a bad reputation from client departments, is that there's a certain amount of feeling superior. You know, we're gossiping about a client department. (Analyste, SCT)

Le bavardage malveillant et le dénigrement des ministères ne doivent pas être considérés qu'au premier degré. Il semble n’y avoir aucune méchanceté derrière cette "pratique», comme s'en défend une participante:

I disagree with that perception completely. Just to demystify - that is not at all the way we speak about or treat departments bere at PCO. And I can confirm that across the board. (...) It is very collaborative and if we have to expose an issue, it's for the sake of the government, not because we want to put a department in the hot seat on purpose. Do you know what I mean? (...) I mean I think that is such a bizarre perception of departments to bave of what the center thinks of them, 'cause it's not true at all. (Analyste, BCP) 
Durant l'échange, cette analyste reconnaît toutefois que lors de conversations à l'interne, il peut arriver que les analystes centraux soient moins tendres à l'égard de leurs clients ministériels. Cette timide admission semble difficilement s'harmoniser avec l'idée que cette participante se fait de sa propre éthique professionnelle, et sans aucun doute, avec le code des valeurs et de l'éthique de l'organisme où elle évolue.

Je retiens ici que les cultures organisationnelles et les responsabilités mêmes qui incombent aux analystes centraux semblent les prédisposer à poser un regard critique sur les ministères. Plus généralement, le commérage apparâ̂t constituer un important "lubrifiant" social. Or, il semble que la culture de prestige ou d'excellence des organismes centraux tend à exacerber cette pratique, et inversement.

However, I think it's just nature. Nobody likes to have their work criticized. And the work of a program analyst is to criticize somebody else's work, and we're encouraged to be as picky as possible, and as exacting as possible. The comments are really where this kind of superiority comes out too; we read sections of submissions out to each other, shaking our heads over the grammar and the frequent idiocy of what's on paper. It's just amazing what makes it into the submission, when we consider that all the people you're dealing with seem to be perfectly competent, but the written document is awful. (Analyste, SCT)

Le sentiment de supériorité dont témoignent les analystes et le dénigrement envers les ministères qui en est le corollaire contribuent à la constitution et au maintien d'un esprit de corps, en même temps qu'ils renforcent le caractère idiosyncratique de l'expérience au sein des organismes centraux.

Cela dit, l'adhésion à la culture organisationnelle n'est ni automatique ni garantie. La même analyste, après plusieurs années passées au centre, demeure très mitigée face à la culture organisationnelle, sentiment qu'elle exprime sans ambages:

The goals, the values of the central agency have never been ones that really resonate with me. That kind of prestige, the search for prestige, that sense of self-importance, I've never really taken it fully on board. (Analyste, SCT)

Les expériences au centre ne sont pas toutes fructueuses, malgré les efforts considérables déployés pour s'adapter à la culture organisationnelle. 


\subsection{Influence et asymétrie dans les rapports}

De façon concomitante, ces cultures organisationnelles évoquent plus concrètement la construction d'une asymétrie dans les rapports avec les ministères, asymétrie qui sous-entend une influence considérable des analystes. Avec leurs partenaires ministériels, les analystes sont appelés à faire fi de la logique bureaucratique en interagissant avec des acteurs hiérarchiquement supérieurs.

One of my contacts at one of the departments is a DG. That's just who I call when I have a question. I think, in that sense, you have connections to bigher levels, being at Finance, that you probably wouldn't have if you weren't in a central agency. (Analyste, FIN)

Les partenaires ministériels avec qui j'ai échangé ne semblaient pas faire grand cas de cette situation:

No, it's the outcome that I look for. Even within the department, if I have to deal with a manager to get something done, I'm happy to do that. But, again at a central agency it's a bit different. If you have a good PCO analyst, it doesn't matter what level they are. (Intervenante ministérielle)

Encore ici, on tend à expliquer un tel modus operandi par l'impératif d'efficacité. Et puisque cette convention se légitime par des motivations "louables", soit faciliter l'échange d'information interministériel pour l'avancement des dossiers, les cadres ministériels ne sauraient s'y opposer. D'où cet accent mis sur le résultat: utilisons les moyens qui permettront d'atteindre les objectifs le plus efficacement possible, quitte à aller à l'encontre de certains des principes régissant l'organisation bureaucratique. Je suppose que le pouvoir d'influence des analystes doit également servir cet impératif d'efficacité, comme l'illustre ce passage: Mostly I would say we're talking to directors and their managers. So mostly my counterpart would be a manager at a client department. But if I wasn't getting what I needed from a manager, I would have to go over

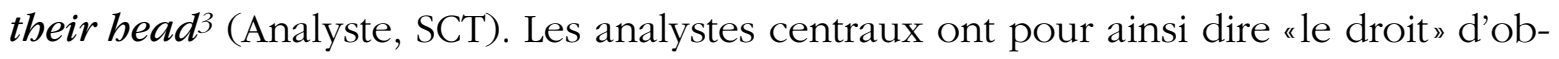
tenir ce qu'ils veulent. D'aucuns liraient, dans cet extrait, l'outrecuidance de ces analystes du centre. Et peut-être y en a-t-il effectivement qui abusent de cette influence conventionnelle. Ils semblent toutefois motivés par autre chose: se faire l'instrument de quelque chose de plus grand qu'eux, un peu comme l'envisageait Weber avec l'idéal-type de la bureaucratie rationnelle-légale.

3. L'utilisation du gras dans les passages cités indique l'emphase ajoutée par l'auteur. 
On représente également le centre comme un lieu où l'on peut véritablement influencer les dirigeants et la classe politique. Ces analystes ont la conviction d'appuyer le bon fonctionnement du gouvernement fédéral — peut-être ont-ils besoin de cette légitimation pour exercer leur influence.

You feel connected to a larger whole. And for me that feeling of having purpose as a central agency analyst, like writing for ministers and, early on, hearing what the [Treasury] Board had to say about the files we were bringing to them. Feeling connected to the machinery of government, decision-making processes, the political arm. (Analyste, SCT)

Toujours à quelques clics du clavier des grands décideurs politiques, les analystes centraux se doivent d'être d'excellents communicateurs. C'est par leurs écrits que se transmettent les recommandations, les avis, les notes et les mises à jour sur l'un ou l'autre des dossiers de l'heure. L'information, celle qu'ils recueillent des ministères, celle qui ruisselle des réunions exécutives et des comités parlementaires, celle qui émerge de leurs propres recherches et analyses, et celle qui se forge de leurs intuitions, de ce qu'ils entendent et observent, toute cette information constitue leur pouvoir d'influence, pouvoir qu'ils doivent user de façon stratégique et parcimonieuse.

I don't abuse it certainly. I don't use briefing up as a right, it's a tool I use when Ifeel like I'm not making progress or I'm not getting through. But it's not something I abuse on a daily basis - otherwise, your audience is no longer going to take you seriously. So you only brief up in a specific way, when you really aren't getting through. (Analyste, BCP)

Certains des analystes ont associé cette influence et la capacité d'action qui en résulte à la volonté de servir la population, voire à appuyer les institutions démocratiques:

But as you move up in your career and you realize the importance of the functions of central agencies to the functioning of democracy and the entire notion of government, it's no longer scary. You recognize the value. (Intervenante ministérielle)

I actually felt close to Canadians. I felt responsible to Canadians. I felt like a representative of the bureaucracy in a good way. I felt important; not personally important, like "I'm important". What I felt is I'm serving an important role that I believe in (...). (Analyste, SCT) 


\section{Discussion}

\subsection{Le centre : représentations d'un milieu gouvernemental spécifique}

De ces représentations émerge une mosaïque bigarrée, à la fois riche et évocatrice des organismes centraux. Généralement méconnus, ceux-ci apparaissent de prime abord comme des organisations occultes aux vastes prérogatives et à l'autorité irréfutable. Ce mystère doublé d'un pouvoir manifeste fascine initialement les participants. Cette conception première, qui n'est pas dénuée d'un certain romantisme, laisse place à des représentations plus nuancées des réalités du centre, dans lesquelles priment des considérations plus concrètes liées à la charge de travail, habituellement très lourde, au rythme d'exécution, proprement effréné, au roulement des employés et à la nature des dossiers et des enjeux, à la fois stratégique et critique. À travers leur discours et leurs récits, les participants décrivent un milieu de travail foncièrement solitaire, que les réalités et les considérations politiques rattrapent facilement, et où le roulement élevé des employés peut parfois entraîner une ambiance plutôt impersonnelle même si, dans certains cas, l'arrimage des personnalités peut contribuer à un environnement convivial, certes, mais toujours en évolution.

Les rôles de coordination, de surveillance, voire de contrôle, que sont appelés à jouer les analystes tendent à façonner leurs relations avec les ministères d'exécution. Ces dernières sont de coutume marquées par la suspicion, le mépris, l'incompréhension ou la défiance. Néanmoins, les mandats des organismes centraux tendent à susciter de véritables vocations chez les analystes, ce qui peut renforcer un certain retranchement dans la culture organisationnelle, et plus généralement, un sentiment de supériorité et de prestige qui colore, à tort ou à raison, les interactions du centre avec ses partenaires ministériels.

\subsection{L'identité : au cœur de la construction sociale du centre}

Il a été question plus haut des divers niveaux d'analyse de la construction identitaire et des dynamiques de récursivité qui les lient. Les récits d'expérience des participants témoignent de ces glissements d'un niveau identitaire à l'autre. Dans l'espace d'une même phrase, ces derniers peuvent passer du "je» au "nous", revenir au "je», puis déclarer "qu'il s'agit». Il y a, dans les discours, un dialogue incessant entre les divers niveaux de construction d'identité, comme l'illustre le prochain extrait, dans lequel une analyste réfléchit à la relation centre-ministères: 


\section{Tableau 1. Construction de sens et niveaux identitaires}

\begin{tabular}{|l|l|l|}
\cline { 2 - 3 } & \multicolumn{1}{|c|}{ Extrait } & \multicolumn{1}{c|}{ Niveau identitaire } \\
\cline { 2 - 3 } Récursivité & $\begin{array}{l}\text { For me I guess the longer I'm at TBS, the more } \\
\text { respect I have for departments, because I'm } \\
\text { simply learning more about each department. } \\
\text { I go deeper into the files. I am not as dismissive } \\
\text { of poor work, you know, or things that don't } \\
\text { make sense because I'm getting a better sense } \\
\text { of what we contribute to that happening, from a } \\
\text { departmental perspective. It's very, I think } \\
\text { there's a tendency when you first start to be very, } \\
\text { to feel more superior, and you're encouraged to } \\
\text { feel that way. lanalyste, SCT) }\end{array}$ & 1 \\
& 1 \\
& 1 \\
\end{tabular}

Mobilisant sa réflexivité, cette analyste aborde son expérience d'abord au «je», puis signale son appartenance au groupe en utilisant le "nous", pour signifier une manière d'être et une conduite propres au groupe, avant de conclure avec ce qu'elle considère être une tendance organisationnelle contraignant les acteurs individuellement. Cet extrait constitue une excellente illustration de la récursivité du processus de construction identitaire: la dernière phrase, qui aborde la subjectivité générique, permet de comprendre la conduite individuelle passée de l'analyste, alors qu'elle était une recrue, conduite dont elle se distancie au présent, dans la première phrase. Sur le fond, on devine une posture identitaire très nuancée, attribuable à plusieurs années d'expérience au centre. L'analyste admet avoir plus de respect envers les ministères depuis qu'elle prend le temps d'approfondir davantage ses dossiers, ce qui l'amène en retour à adopter une attitude plus compréhensive envers ses interlocuteurs. Cet extrait montre ainsi que l'acteur peut contribuer à façonner l'identité organisationnelle en ajustant sa conduite, c'est-à-dire en déconstruisant et en reconstruisant le sens de ses actions. Bien sûr, si ce processus demeure intrasubjectif, il s'agira ni plus ni moins d'une rafraîchissante exception à la norme de conduite envers les interlocuteurs ministériels. Néanmoins, autant l'organisation a la capacité de contraindre et d'habiliter les acteurs, autant ces derniers peuvent agir réflexivement dans la construction sociale des identités, la leur et celle de l'organisation.

Le recours au "je», au "nous», au "c'est» et au "tu» appuie le processus de différenciation auquel se livrent les analystes centraux, individuellement et collectivement. L'utilisation du "nous» sous-entend dès lors un sentiment d'appartenance au groupe, ce qui dénote l'émergence d'une identité sociale. L'adhésion à un groupe confère un caractère distinctif à l'organisation, qui se manifeste, dans le discours des participants, 
par l'opposition de ce «nous» à "eux» ou à «ils». La reconnaissance de ce qui différencie les analystes centraux de leurs interlocuteurs ministériels consacre l'identité sociale et cristallise les caractéristiques spécifiques des organismes centraux.

Ce passage met par ailleurs en lumière le potentiel heuristique de la phénoménologie herméneutique comme méthode de compréhension et moyen de connaître. Dans ces quelques lignes, l'analyste pose un regard nouveau sur des actions passées, proposant une réinterprétation de ces dernières et insufflant du sens dans sa conduite actuelle, sens qui orientera également ses actions futures. Ce moment réflexif et rétrospectif est la clef de voûte de la phénoménologie herméneutique.

L'identité organisationnelle, en tant que construction sociale, est donc un processus intersubjectif et cognitif visant à marquer, à étiqueter et à objectiver les schémas d'interprétation communs des acteurs (Haslam, Cornelissen, et Werner, 2017, p. 321). Les analystes effectuent collectivement un exercice de cadrage par lequel ils élaborent diverses représentations de l'organisation. Ces dernières parlent autant d'eux que de l'organisation, rendant leur expérience significative (2017, p. 322).

\section{Conclusion}

De ce portrait foisonnant et complexe issu de la collision des représentations multiples du centre émerge plusieurs éléments fort pertinents pour comprendre l'expérience des analystes au sein des organismes centraux: les sentiments de supériorité et de prestige associés à l'expérience au sein des organismes centraux, la culture de dénigrement des ministères d'exécution, la posture rationnelle et objective des analystes ainsi que leur pouvoir d'influence. Si ces éléments sont constitutifs des processus de socialisation organisationnelle et de construction identitaire vécus individuellement et collectivement, ils laissent également entrevoir la construction sociale des organismes centraux. En délaissant l'examen structuro-fonctionnaliste au profit des acteurs et de leur démarche identitaire, on devine des organisations très sélectives, exigeantes envers le personnel et peu enclines à l'empathie. Si le travail au centre suscite la fierté et tient de la vocation noble, il s'appuie sur une injonction au dépassement de soi par l'efficacité, l'efficience et un dévouement physique et psychique sans pareil dans la fonction

publique fédérale. Certains y devineront une certaine violence organisationnelle se manifestant par une déshumanisation des rapports humains et l'intransigeance des milieux de travail. 


\section{Bibliographie}

Annink, A. (2017). Using the Research Journal during Qualitative Data Collection in a Cross-Cultural Context. Entrepreneurship Research Journal, 7(1), 223-217.

Ashforth, B. E., Rogers, K. M., et Corley, K. G. (2011). Identity in Organizations: Exploring Cross-Level Dynamics. Organization Science, 22(5), 1144-1156.

Barley, S. R., et Tolbert, P. (1997). Institutionalization and structuration: Studying the links between action and institution. Organization Studies, 18(1), 93-117.

Bernier, L., et Fortier, A. (2014). Les organismes centraux. Dans A.-G. Gagnon, D. Sanschagrin, et L. Bernier (dir.). Politique québécoise et canadienne: une approche pluraliste (p. 239-261). Québec: Les Presses de l'Université du Québec.

Boje, D. M. (2003). Using Narrative and Telling Stories. Dans D. Holman et R. Thorpe (dir.). Managing and Language: the Manager as a Practical Author (p. 41-53). London: SAGE.

Brown, A. D. (2017). Identity Work and Organizational Identification. International Journal of Management Reviews, 19(3), 296-317.

Campbell, C., et Szablowski, G. J. (1979). The Superbureaucrats: Structure and Behaviour in Central Agencies. Toronto: MacMillan.

Canada. (2017). Groupe économique et services de sciences sociales (EC) - Norme d'évaluation des emplois. Repéré à https://www.canada.ca/fr/secretariat-conseil-tresor/services/conventionscollectives/evaluation-emplois/groupe-economique-services-sciences-sociales-norme-evaluationemplois.html

Chia, R. (1996). The Problem of Reflexivity in Organizational Research: Towards a Postmodern Science of Organization. Organization, 3(1), 31-59.

Clark, I. D. (1994). Restraint, renewal, and the Treasury Board Secretariat. Canadian Public Administration, 37(2), 209-248.

Clark, I. D., et Swain, H. (2005). Distinguishing the real from the surreal in management reform: suggestions for beleaguered administrators in the government of Canada. Canadian Public Administration, 48(4), 453-476.

Dunn, C. J. C. (2010). The central executive in Canadian government: Searching for the Holy Grail. Dans C. J. C. Dunn (dir.), The Handbook of Canadian Public Administration (2e éd.). Don Mills: Oxford University Press.

Giddens, A. (1987). La constitution de la société: éléments de la théorie de la structuration. Paris: Presses universitaires de France.

Hart, J. (2014). Central Agencies and Departments: Empowerment and Coordination. Dans B. G. Peters et D. J. Savoie (dir.). Taking stock: assessing public sector reforms (p. 285-309). Montréal: McGill-Queen's University Press.

Haslam, S. A., Cornelissen, J. P., et Werner, M. D. (2017). Metatheories and Metaphors of Organizational Identity: Integrating Social Constructionist, Social Identity, and Social Actor Perspectives within a Social Interactionist Model. International Journal of Management Reviews, 19(3), 318-336. 
Haslam, S. A., et Ellemers, N. (2005). Social identity in industrial and organizational psychology: Concepts, controversies and contributions. International review of industrial and organizational psychology, 20(1), 39-118.

Hicks, M. (1973). The Treasury Board of Canada and its clients: five years of change and administrative reform 1966-71. Canadian Public Administration, 16(2), 182-205.

Jun, J. S. (2006). The social construction of public administration: interpretive and critical perspectives. Albany: State University of New York Press.

Knights, D., et Clarke, C. (2017). Pushing the boundaries of amnesia and myopia: a critical review of the literature on identity in management and organization studies. International Journal of Management Reviews, 19(3), 337-356.

Lindquist, E. (1996). New Agendas for Research on Policy Communities: Policy Analysis, Administration, and Governance. Dans L. Dobuzinskis, M. Howlett, et D. Laycock (dir.). Policy Studies in Canada: The State of the Art (p. 219-241). Toronto, Buffalo, London: University of Toronto Press.

Lutgen-Sandvik, P. (2008). Intensive Remedial Identity Work: Responses to Workplace Bullying Trauma and Stigmatization. Organization, 15(1), 97-119.

Mead, G. H. (1934). Mind, Self and Society. Chicago, IL: University of Chicago Press.

Potter, E. H. (2000). Treasury Board as a Management Board: The Re-Invention of a Central Agency. Dans L. A. Pal (dir.), How Ottawa spends, 2000-2001: Past Imperfect, Future Tense (p. 95-129). Don Mills, On: Oxford University Press.

Robertson, G. (1971). The changing role of the Privy Council Office. Canadian Public Administration, 14(4), 487-508.

Savoie-Zajc, L. (2009). L'entrevue semi-dirigée. Dans B. Gauthier (dir.), Recherche en sciences sociales. De la problématique à la collecte des données (p. 337-359). Québec: Presses de l'Université du Québec.

Savoie, D. (1997). Le rôle des organismes centraux au sein du gouvernement du Canada. Dans J. Bourgault, M. Demers, et C. Williams (dir.), Administration publique et management public: expériences canadiennes (p. 59-69). Québec: Les Publications du Québec.

Savoie, D. (1999). Governing from the centre: the concentration of power in canadian politics. Downsview, On: University of Toronto press.

Schick, A. (2001). The changing role of the central budget office. OECD Journal on Budgeting, 1(1), 9-26.

Sloan, A., et Bowe, B. (2014). Phenomenology and hermeneutic phenomenology: the philosophy, the methodologies, and using hermeneutic phenomenology to investigate lecturers' experiences of curriculum design. Quality \& Quantity, 48(3), 1291-1303.

Sluss, D. M., et Ashforth, B. E. (2007). Relational identity and identification: Defining ourselves through work relationships. Academy of management review, 32(1), 9-32.

Tajfel, H. (1974). Social identity and intergroup behaviour. Social Science Information, 13(2), 65-93. 
Veilleux, G., et Savoie, D. (1988). Kafka's castle: The Treasury Board of Canada revisited. Canadian Public Administration, 31(4), 517-538.

Watson, T. J. (2009). Narrative, life story and manager identity: A case study in autobiographical identity work. Human Relations, 62(3), 425-452.

White, G. (2005). Cabinets and first ministers. Vancouver: University of British Columbia Press.

White, J. D. (1999). Taking language seriously: the narrative foundations of public administration research. Washington, D.C.: Georgetown University Press.

Willig, C. (2001). Interpretative phenomenology. Dans C. Willig (dir.). Introducing qualitative research in psychology: adventures in theory and method (p. 50-69). Maidenhead, Berkshire, England: Open University Press. 